\title{
黄土丘陵区不同立地条件下猪毛蒿种内、 种间竞争
}

\author{
杜 峰 梁宗锁* 山 仑 陈小燕 \\ (西北农林科技大学中国科学院水利部水土保持研究所, 土壤侵蚀与旱地农业国家重点实验室 陕西杨凌 712100)
}

\begin{abstract}
摘 要 猪毛蒿 (Artemisia scoparia) 通常是黄土丘陵区撂荒演替前期群落优势种, 在无人为干扰的情况下 猪毛蒿群 落通常会向冰草 (Agropyron cristatum) 群落或阿尔泰狗娃花 (Heteropappus altaicus) 群落 或长芒草 ( Stipa bungeana) 群落 等演替。该文通过河阶地和梁峛阴坡中猪毛蒿生长特征的调查和种内、种间竞争田间试验，从植物竞争角度对猪 毛蒿群落的演替机制给予了解释。结果表明:1)两类样地中猪毛蒿的生长都趋于小型化, 有少数大个体和多数小 个体, 都存在异速生长现象, 说明两类样地存在竞争, 且对猪毛蒿的生长形态具有塑造作用;2) 由于单位地上生物 量竞争效应排除了立地条件和个体大小的影响，因而比总竞争效应更能说明种对间的相对竞争能力。梁峛阴坡地 和一、二级河阶地三种立地条件下, 7 种测试植物中对猪毛蒿的相对竞争能力以演替后期多年生植物较高, 而演替 前期一年生植物较低，说明演替后期种对前期种的竞争抑制是演替驱动力之一；3) 以各测试植物对猪毛蓄单位重 量竞争抑制程度平均值来看，以梁峁阴坡地最大，二级河阶地次之，一级河阶地最小，说明立地条件越差，土壤资源 可利用水平越低, 竞争越激烈; 4)一级河阶地和梁峁阴坡地各测试植物对猪毛蒿的竞争等级发生了显著变化, 说明 环境条件差别较大时 植物的竞争等级会发生变化。
\end{abstract}

关键词 猪毛蓄 种间竞争 种内竞争 异速生长

\section{INTRASPECFIC AND INTERSPECFIC COMPETITION OF ARTEMISIA SCOPARIA UNDER DIFFERENT SITE CONDITIONS IN THE HILLY REGION OF LOESS PLATEAU}

\author{
DU Feng LIANG Zong-Suo* SHAN Lun and CHEN Xiao-Yan
}

(Institute of Soil and Water Conservation, Chinese Academy of Sciences , Ministry of Water Resource, State Key Laboratory of Soil Erosion and Dryland Farming on Loess Plateau , Northwest Sci-Tech University of Agriculture and Forestry, Yangling, Shaanxi 712100 , China)

\begin{abstract}
Background and Aims In hilly Loess region, Artemisia scoparia is always the dominator in the early abandoned field communities. It may be replaced by Agropyron cristatum or Heteropappus altaicus or Stipa bungeana in next succession stage when no disturbance occurs. In order to study the direction of Artemisia scoparia community succession under different standing conditions, we conducted transplant experiments to test the intraspecfic and interspecfic competition of Artemisia scoparia in river terrace and northern mound land and , at the same time, its morphological traits were investigated.
\end{abstract}

Methods In the experiments, nine species (three coexisting species, five later sere species and Artemisia scoparia itself) were chosen and transplanted as test species, but due to the low survival of two species, only seven species were analyzed to test the relative competition ability to phytometer Artemisia scoparia under different standing conditions.

Key Results The results show that the individuals tend to be miniaturized , i.e. , its communities are composed mainly of small-sized individuals and competition can change the morphological traits. The unit biomass competition intensity is better than total competition intensity in explaining plants' relative competitive ability when local conditions and plant size are considered. Under the northern mound land and the lowest river terrace , late-successional and perennials species have higher competitative ability than early-successional and annual plants , suggesting that the competition of late- against early-successional species is one of the driving force to succession. Comparison of the competition between the seven test species and the unit biomass of Artemisia scoparia , the northern mound land ranks the first, lower river terrace the second and the lowest terrace the 
third , suggesting that intensive competition occurs more severely in poor growing conditions. There is significant difference in the ability of competition between the test species and Artemisia scoparia from the lowest river terrace to the northern mound land, suggesting that the ranks of the competition ability may vary depending on standing conditions.

Conclusions As competition is one of the decisive factors in constructing plant community , the shift or variation of competition ability between coexisting species and sere species may result in different community structure and dynamics, so it changes the direction and pathway of succession under different standing conditions.

Key words Artemisia scoparia , Interspecific competition , Intraspecfic competition , Allometry

竞争是两个以上有机体间存在的负相互作用 (李博等, 1998)，在共享资源有限的情况下竞争会导 致个体生长量、存活率和繁殖率的降低 (Begon et $a l .1990)$; 此外竞争也是一个资源间接调节的过 程, 是植物互相对资源吸收与利用从而引起资源波 动及植物对资源波动的响应与反应过程 (Goldberg， 1990)。因此竞争可以采用生理指标 (如资源吸收利 用量或利用效率、气孔导度或水势等) (Hamiluon et $a l$. 1999)或生态指标(如高度, 地上、地下生物量, 相对生长速率,营养面积等) (Aguiar et al.,2001; Chen 2001; Leuschner et al. ,2001; 李仲芳和王刚， 2002 ;Tielbörger \& Kadmon,2000;王政权等,2000;刍 春静和徐文铎, 1998) 来研究 (张泽浦等,2000)。竞 争是群落组成、结构与动态的决定性因素 (Aerts， 1999 ;Martin et al . 2001;Tilman ,1988) 演替后期种 对前期种较强的竞争是植被演替的动力之一 (Fowler, 1986; Negbi \& Evenari ,1961)。植被动态的长 期研究结果表明竞争对群落演替的作用主要有两个 机制 演替早期物种繁殖力高, 繁殖体传播距离远, 先定居生境, 但竞争力弱, 后期物种相反, 这种演替 机制生态学家称做定居-竞争权益法则 (Colonizationcompetition tradeoff)。即使演替前期与后期物种同 时出现，因演替前期物种在资源相对丰富的条件下 生长迅速, 短期内要比演替后期物种表现好, 以后随 资源的消耗, 生长变慢且存活率降低, 演替后期物种 竞争力强, 在资源较低时能生长、存活和竞争, 在无 干扰的条件仍能代替演替前期物种成为群落中的优 势种, 这种机制称做演替生态位 (Succession niche) (Rees et al. 2001)。

由于竞争在个体形态塑造和群落组成、结构与 动态方面的重要意义，因而受到了越来越多的关注， 但生态学家对一些重大生态学问题存在长期的争 论, 如植物竞争等级是固定的还是随机的, 竞争不对 称性是否随着环境生产力的增加而增加, 竞争在环 境生产力梯度上对群落动态的相对重要性等 (杜峰 等 ,2004)。为此本文进行了不同立地条件下猪毛蒿
(Artemisia scoparia) 的种内、种间竞争试验研究, 试图 回答环境梯度上几种植物对猪毛蒿竞争的变化规 律 ,并解释猪毛蒿群落的演替方向。

\section{1 自然概况及研究方法}

\section{1 研究地点自然概况}

研究地点位于陕北黄土高原丘陵沟壑区的安塞 县高桥乡, 年平均日照时数为 $2300 \sim 2570 \mathrm{~h}$, 年平 均日照总辐射量为 $499.4 \sim 555.2 \mathrm{~kJ} \mathrm{~cm}$ ch $^{-2}$, 年平均 降水量在 $490.5 \sim 663.3 \mathrm{~mm}$ 之间, 其中 $6 \sim 8$ 月降雨 量占全年降水的 $60 \% \sim 80 \%$, 年平均气温在 $7.7 \sim$ $10.6{ }^{\circ} \mathrm{C}$ 之间, 无霜期为 $142 \sim 175 \mathrm{~d}$ 左右, 平均 157 $\mathrm{d}$,干燥度 $1.2, \geqslant 0{ }^{\circ} \mathrm{C}$ 积温 $3733.1{ }^{\circ} \mathrm{C}, \geqslant 10{ }^{\circ} \mathrm{C}$ 积温 $3170.3{ }^{\circ} \mathrm{C}$ 地带性土壤为黄绵土。

试验样地为 3 块, 分别为仁台沟一级、二级河阶 地 $\left(109^{\circ} 16^{\prime} 15.3^{\prime \prime} \mathrm{E}, 36^{\circ} 39^{\prime} 9.5^{\prime \prime} \mathrm{N}\right.$, 海拔分别为 1080 和 $1090 \mathrm{~m}$ ) 和千腰岘梁峁阴坡地 $\left(109^{\circ} 12^{\prime} 58.5^{\prime \prime} \mathrm{E}\right.$, $36^{\circ} 40^{\prime} 02.7^{\prime \prime} \mathrm{N}$, 海拔 $1270 \mathrm{~m}$ ) ,样地面积大小分别 为 $890 、 1320$ 和 $4560 \mathrm{~m}^{2}$ 。其中一级河阶地距离水 源垂直距离为 $3 \mathrm{~m}$, 水平距离为 $5 \sim 20 \mathrm{~m}$, 群落主要 种有长芒草 (Stipa bungeana)、萎陵菜 (Potentilla chinensis)、二列叶萎陵菜 (Potentilla bifurca)、艾蒿 (Artemisia argyi) 和蒲公英( Taraxacum mongolicum) 等 35 种。二级河阶地距离水源垂直距离为 $10 \mathrm{~m}$, 水平 距离为 $30 \sim 60 \mathrm{~m}$, 群落主要种有铁杆蓄 (Artemisia sacrorum) 、芠蓠 (Artemisia giraldii)、硬质早孰禾( Poa sphondylodes)、白羊草 (Bothriochloa ischaemum)、中华 隐子草 (Cleistogenes chinessis)、冰草 (Agropyron cristatum)、硬皮憼 (Allium ledebourianum) 和阿尔泰狗娃花 (Heteropappus altaicus) 等 22 种。千腰岘梁峁阴坡地 距离水源 $600 \mathrm{~m}$, 群落主要物种有猪毛蕎、苦苣 (Sonchus oleraceus)、苣卖菜( Sonchus arvensis)、刺儿菜 (Artemisia scoparia)、猪毛菜 (Salsola collina)、狗尾草 (Setaria viridis) 和沙蓬 (Argiophyllum arenarium) 等 13 种。

猪毛蒿特征调查样地为新庄科一级河阶地 
$\left(109^{\circ} 11^{\prime} 36.0^{\prime \prime} \mathrm{E}, 36^{\circ} 39^{\prime} 00.6^{\prime \prime} \mathrm{N}\right.$, 海拔 $\left.1090 \mathrm{~m}\right)$ 和千 腰岘梁峛阴坡地, 样地面积大小分别为 580 和 4560 $\mathrm{m}^{2}$ 。其中新庄科一级河阶地距离水源水平距离为 $5 \sim 30 \mathrm{~m}$, 重直距离为 $2.5 \mathrm{~m}$, 土壤耕作层及以下有粗 砾石, 质地较粗, 群落主要物种组成为猪毛蒿、黄花 蒿( Artemisia annual) 等 23 种。

\section{2 研究方法}

\subsection{1 猪毛蒿生长特征调查}

在新庄科一级河阶地和千腰岘梁峛阴坡地上沿 $\mathrm{S}$ 形每隔 $1.5 \mathrm{~m}$ 测量半径为 $50 \mathrm{~cm}$ 样圆内所有猪毛 蕎的高度与地上生物量, 并记录群落的总盖度和分 种盖度。调查时间为 2002 和 2003 年 6 月上旬至 9 月上旬, 河阶地和梁峁阴坡地共调查 24 和 22 个样 圆 猪毛蒿个体数共 256 和 182 株。

\subsection{2 田间竞争试验}

在千腰岘梁峁阴坡地和仁台沟一级、二级河阶 地上进行两年的移栽试验，以测试不同立地条件下 演替后期群落主要种对猪毛蒿的种间竞争, 演替同 一阶段群落共存种对猪毛蒿的种间竞争, 及猪毛蒿 的种内竞争。移栽时间为 2002 和 2004 年的 5 月 1 日至 15 日。先将样地上的所有植物手工去除, 然后 在摞荒地上挖一系列大小为 $20 \mathrm{~cm} \times 20 \mathrm{~cm}$ 深为 30 $\mathrm{cm}$ 的移栽穴 移栽穴间距离 $1.5 \mathrm{~m}$ 。从邻近草地上 挖取大小均匀一致的竞争测试植物 (Test species), 将测试植物按正方形排列方式移栽于四角, 目标种 (Phytometer)猪毛蒿移栽于穴中心, 无竞争对照为目 标种单独移栽。移栽完毕后浇水, 移栽一月左右待 植物成活进入正常生长期后，手工去除移栽穴内外 非试验植物和周围所有植物。测试植物 9 种, 其中 :
猪毛蓠共存种有猪毛菜、狗尾草、刺儿菜; 演替后期 主要种有阿尔泰狗娃花、长芒草、中华隐子草、硬质 早孰禾、白羊草 种内竞争测试植物为猪毛蒿。梁崩 阴坡样地因不具备浇水条件, 每年设计重复 20 次以 保证成活率, 共计 $20 \times 9$ 穴。一级和二级河阶地每 年设计重复 10 次，共计 $10 \times 9$ 穴。2002 和 2004 年 9 月下旬至 10 月上旬进行竞争响应变量植株高度和 地上生物量鲜重的测定。

对重复次数在 8 穴以上的数据进行相对竞争强 度的计算, 其中猪毛菜、刺儿菜、硬质早熟禾和二级 河阶地中的白羊草因成活率低, 重复次数少, 未进行 数据处理。

土壤水分环境背景值为 2004 年生长旺盛期 3 次测定的平均值, 测定方法为土钻烘干法。土壤养 分在 2004 年生长期末按 S" 形取土样 6 个。测定方 法 :土壤有机质— $\mathrm{K}_{2} \mathrm{Cr}_{2} \mathrm{O}_{7}$ 浓硫酸外加热法; 全氮 一凯氏定氮法;速效氮——碱解扩散法;速效磷 $-\mathrm{NaHCO}_{3}$ 法; 速效钾— $-\mathrm{NH}_{4} \mathrm{OAc}$ 法 + 火焰光度 法(表 1)。

\section{3 数据处理}

\subsection{1 异速生长的拟合}

猪毛蒿高度和地上生物量调查数据按 Weiner 和 Thomas(1992)的界定，用下式进行异速生长拟合 (李仲芳和王刚,2002)：

$$
y=a x^{2}+b x+c
$$

其中因变量 $y$ 为高度,自变量 $x$ 为地上生物 量。若拟合结果显著, 说明猪毛蓠的种内、种间竞争 较为激烈，表现为非线形异速生长，否则就用线性回 归, 最后在 $\log H-\log B$ 坐标平面上绘图 其中 $H$ 和 $B$

表 1 研究地点土壤水分、养分概况

Table 1 Summary of soil moisture and nutrition in investigation and experiment sites

\begin{tabular}{|c|c|c|c|c|c|c|c|c|}
\hline \multirow{2}{*}{$\begin{array}{c}\text { 调查和试验地点 } \\
\text { Investigation and experiment sites }\end{array}$} & \multicolumn{3}{|c|}{$\begin{array}{l}\text { 土壤水分含量 }(\%) \\
\text { Soil moisture content }\end{array}$} & \multicolumn{5}{|c|}{ 土壤养分 Soil nutrients } \\
\hline & $0 \sim 20 \mathrm{~cm}$ & $20 \sim 40 \mathrm{~cm}$ & $40 \sim 60 \mathrm{~cm}$ & $\begin{array}{c}\text { 有机质 } \\
\text { Organic } \\
\left(\mathrm{g} \mathrm{kg}^{-1}\right)\end{array}$ & $\begin{array}{c}\text { 全氮 } \\
\text { Total N } \\
\left(\mathrm{g} \mathrm{kg}^{-1}\right)\end{array}$ & $\begin{array}{c}\text { 速效氮 } \\
\text { Active N } \\
\left(\mathrm{mg} \mathrm{kg}^{-1}\right)\end{array}$ & $\begin{array}{c}\text { 速效磷 } \\
\text { Active P } \\
\left(\mathrm{mg} \mathrm{kg}^{-1}\right) \\
\end{array}$ & $\begin{array}{c}\text { 速效钾 } \\
\text { Active K } \\
\left(\mathrm{mg} \mathrm{kg} \mathrm{kg}^{-1}\right)\end{array}$ \\
\hline $\begin{array}{l}\text { 新庄科一级河阶地 } \\
\text { The lowest river terrace in Xinzhuangke }\end{array}$ & 10.55 & 10.12 & 11.15 & 7.86 & 0.77 & 20.36 & 4.89 & 122.16 \\
\hline $\begin{array}{l}\text { 千腰岅梁峁阴坡 } \\
\text { Northern mound land in Qianyaoxian }\end{array}$ & 6.04 & 7.28 & 7.74 & 5.42 & 0.39 & 16.45 & 2.08 & 74.62 \\
\hline $\begin{array}{l}\text { 仁台沟一级河阶地 } \\
\text { The lowest river terrace in Rentaihou }\end{array}$ & 14.76 & 15.20 & 15.83 & 11.25 & 0.63 & 20.21 & 1.66 & 71.01 \\
\hline $\begin{array}{l}\text { 仁台沟二级河阶地 } \\
\text { Lower river terrace in Rentaigou }\end{array}$ & 9.06 & 10.79 & 11.28 & 7.52 & 0.59 & 17.08 & 1.87 & 68.43 \\
\hline
\end{tabular}


分别为高度和生物量。

\subsection{2 相对竞争强度的计算}

按下面 3 式计算各种植物对猪毛蕎总的相对竞 争强度、单位地上生物量竞争强度 (Aguiar et al. , $2001 ;$;Grace ,1995)。

$$
\begin{aligned}
& R C I_{\text {Total }}^{B}=\left(B_{\text {无 }}-B_{\text {有 }}\right) / B_{\text {无 }} \\
& R C I_{T_{\text {otal }}}^{H}=\left(H_{\text {无 }}-H_{\text {有 }}\right) / H_{\text {无 }} \\
& R C I_{\text {Pergram }}=R C I / B_{\text {邻体 }}
\end{aligned}
$$

其中 $R C I$ 为相对竞争强度 (Ralative conpetition intensity）, $R C I_{\text {Total }}$ 和 $R C I_{\text {Pergram }}$ 分别代表总体相对竞争 强度和单位地上生物量竞争强度, $B$ 和 $H$ 代表生物

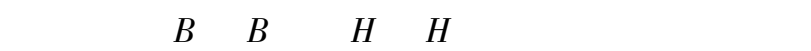
猪毛茣的地上生物量和高度, $B_{\text {邻体 }}$ 为邻体地上生物 量。

使用 Orgin5.0 作图并进行非线形异速生长拟 合。数据处理软件为 STATISTICA6.0 和 DPS 数据处 理系统(唐启义和冯明光,1997)。由于植物高度和 生物量为非正态分布, 因此数据分析使用非参数检 验。其中株高和地上生物量分布使用 $X^{2}$ 检验, 均 值差异显著性使用 Mann-Whitney $U$ 检验, 不同立地 条件竞争差异显著性使用 Kruskal-Wallis 显著性检 验，竞争等级差异显著性使用 Spearman 秩相关检 验。

\section{2 结果与分析}

\section{1 不同立地条件下猪毛蒿的生长特征}

河阶地中单株猪毛蒿的最大生物量和株高分别 为 $269.62 \mathrm{~g}$ 和 $175.0 \mathrm{~cm}$, 最小为 $0.15 \mathrm{~g}$ 和 $1.0 \mathrm{~cm}$, 平 均为 $55.07 \mathrm{~g}$ 和 $84.7 \mathrm{~cm}$ 樑峁阴坡地猪毛蒿的最大
生物量和株高分别为 $101.53 \mathrm{~g}$ 和 $112.8 \mathrm{~cm}$, 最小为 $0.18 \mathrm{~g}$ 和 $2.0 \mathrm{~cm}$, 平均为 $10.5 \mathrm{~g}$ 和 $51.5 \mathrm{~cm}$, 可见在 立地条件较好的河阶地中，猪毛蕎的长势较立地条 件较差的梁峁阴坡上好。根据猪毛蒿生物量的频次 分布图 (图 1), 两种立地条件下猪毛蕎的生长都趋 于小型化, 有较少的大个体和多数小个体。高度和 生物量的频次分布明显不同，以株高作为理论频数， 对地上生物量的观察频数进行 $X^{2}$ 检验, 表明株高 和生物量的频次分布不相符合 $\left(X^{2}\right.$ 检验值远小于 $0.05)$, 说明猪毛蒿的生长存在异速生长, 其地上部 分的生长形态受到来自种内或种间的较强竞争作 用。株高与生物量经非线形异速生长方程拟合，结 果显著, 在 $\log H-\log B$ 平面表现为曲线关系(图 2 与 表 2) ,进一步表明河阶地和梁峁阴坡地的猪毛蒿群 落中存在较强的竞争。

河阶地土壤水分等立地条件较好(表 1)，猪毛 蒿邻体植物有 23 种之多。除猪毛蒿、刺儿菜和黄花 蒿外, 其它都位于下层(平均高度 $38.4 \mathrm{~cm}$ ), 长势较 弱 地上生物量为 $1.28 \mathrm{~kg} \mathrm{~m}^{-2}$ 总密度为 23.68 株 $\mathrm{m}^{-2}$ 其中猪毛蒿、黄花蒿和刺儿菜分别为 13.70 、 2.01 和 1.52 株 $\mathrm{m}^{-2}$, 其它邻体植物为 6.45 株 $\mathrm{m}^{-2}$ (表 2)。梁峁阴坡地立地条件较差, 猪毛茣的伴生 物植物较少, 只有 13 种, 地上生物量为 $0.26 \mathrm{~kg}$ $\mathrm{m}^{-2}$,总密度为 19.42 株 $\mathrm{m}^{-2}$,其中猪毛蒿为 10.60 株 $\mathrm{m}^{-2}$ 。

河阶地猪毛蒿高度和地上生物量变异性 (生物 量和株高相对变异强度分别为 1.61 和 0.57 ) 较梁峁 阴坡地大 (生物量和株高的相对变异强度分别为 1.50 和 0.50$)$ 。

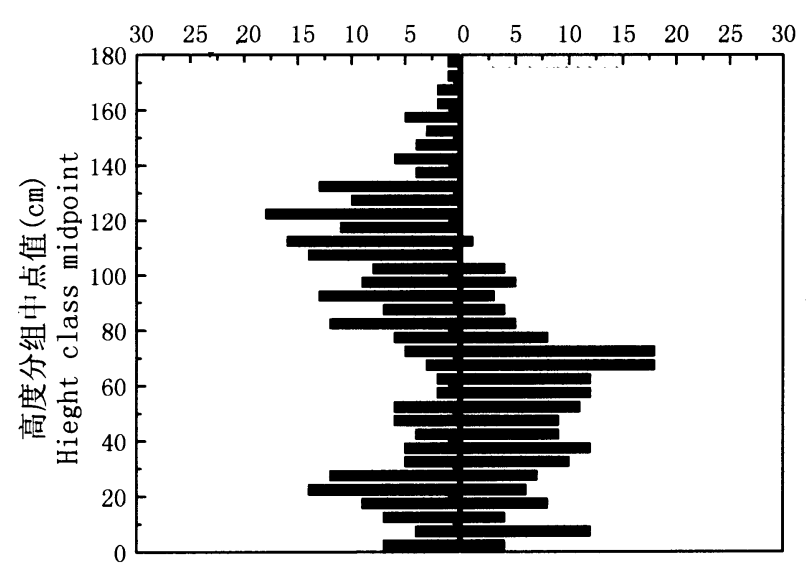

个体数 Number of individuals

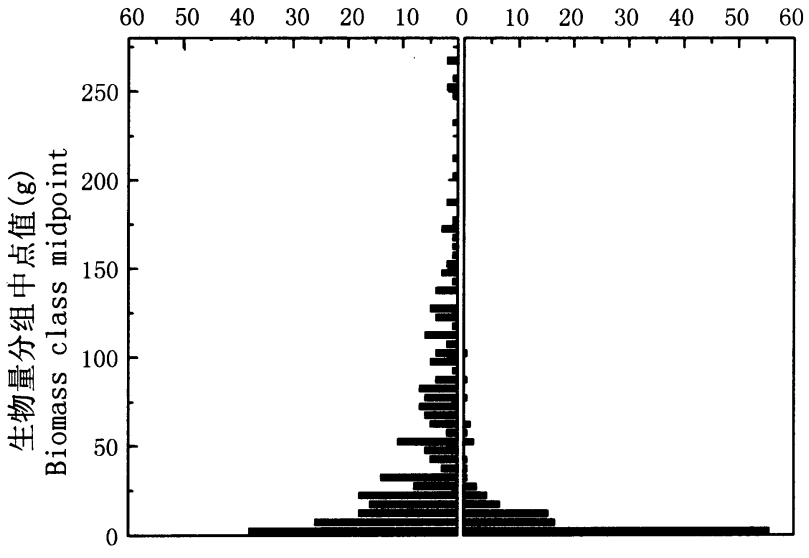

个体数 Number of individuals

图 1 不同立地条件下猪毛蒿高度和地上生物量频次分布图

Fig. 1 Frequency histogram of height and aboveground biomass of Artemisia scoparia under different standing conditions 

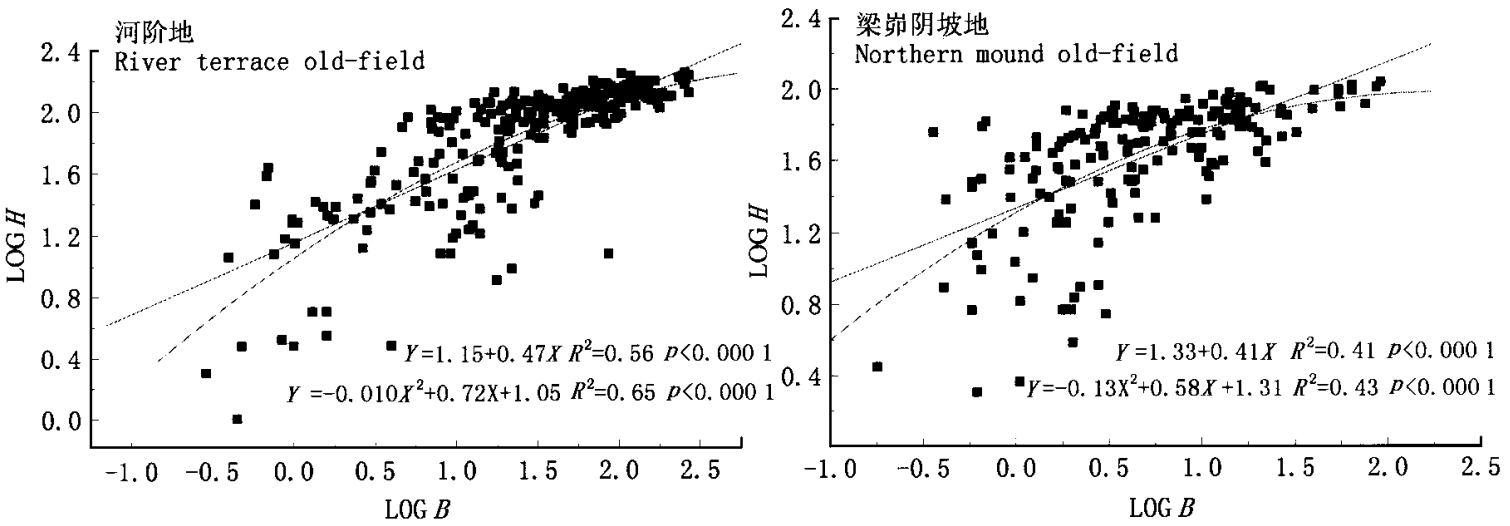

图 2 猪毛蒿非线形异速生长 $\log H-\log B$ 平面图

Fig.2 $\log H-\log B$ graph of nonlinear allometry of Artemisia scoparia

表 2 不同立地条件下猪毛蒿种内、种间竞争及其测试植物的生长特征

Table 2 Growth traits of Artemisia scoparia with and without test species

\begin{tabular}{|c|c|c|c|c|c|c|c|c|}
\hline \multirow{4}{*}{$\begin{array}{l}\text { 地点 } \\
\text { Sites }\end{array}$} & \multicolumn{3}{|c|}{ 测试植物 Test species } & \multicolumn{4}{|c|}{ 目标种 Phytometer } & \multirow{4}{*}{ 样本数 $N$} \\
\hline & \multirow{3}{*}{$\begin{array}{c}\text { 植物种名 } \\
\text { Species }\end{array}$} & \multicolumn{2}{|c|}{$\begin{array}{c}\text { 地上生物量 }(\mathrm{FWg} / \text { 穴) } \\
\text { Aboveground biomass } \\
\text { per bunch }\end{array}$} & \multicolumn{2}{|c|}{$\begin{array}{c}\text { 高度 } \\
\text { Height }(\mathrm{cm})\end{array}$} & \multicolumn{2}{|c|}{$\begin{array}{c}\text { 地上生物量 ( } \mathrm{FWg} \text { /穴) } \\
\text { Aboveground biomass } \\
\text { per bunch }\end{array}$} & \\
\hline & & 平均 & 标准差 & 平均 & 标准差 & 平均 & 标准差 & \\
\hline & & Mean & $S D$ & Mean & $S D$ & Mean & $S D$ & \\
\hline 梁岇阴坡 & 无 No & & & 100.49 & 28.66 & 255.58 & 110.46 & 27 \\
\hline \multirow{7}{*}{$\begin{array}{l}\text { Northern } \\
\text { mound land }\end{array}$} & 狗尾草 Setaria viridis & 6.20 & 1.82 & 94.14 & 27.85 & 238.45 & 99.47 & 15 \\
\hline & 猪毛蒿 Artemissia scoparia & 210.84 & 135.41 & 45.82 & 24.03 & 14.42 & 9.79 & 22 \\
\hline & 冰草 Agropyron cristatum & 2.23 & 2.00 & 98.18 & 20.01 & 212.97 & 86.56 & 25 \\
\hline & 阿尔泰狗娃 Heteropappus altaicu & us 32.54 & 25.53 & 72.11 & 14.67 & 117.3 & 87.66 & 19 \\
\hline & 长芒草 Stipa bungeana & 5.46 & 3.85 & 87.25 & 15.89 & 189.86 & 91.47 & 13 \\
\hline & 中华隐子草 Ceistogenes squarros & $\mathrm{sa} 42.51$ & 26.13 & 42.2 & 15.40 & 60.07 & 30.2 & 16 \\
\hline & 白羊草 Bothriochloa ischaemum & 67.89 & 52.57 & 38.05 & 1756 & 41.24 & 27.38 & 12 \\
\hline 一级河阶地 & 无 No & & & 91.56 & 31.99 & 243.19 & 139.01 & 13 \\
\hline \multirow{7}{*}{$\begin{array}{l}\text { The lowest river } \\
\text { treeace }\end{array}$} & 狗尾草 $S$. viridis & 3.58 & 2.56 & 88.87 & 27.58 & 240.07 & 120.33 & 12 \\
\hline & 猪毛蒿 A. scoparia & 134.25 & 78.89 & 71.26 & 31.07 & 48.36 & 22.52 & 9 \\
\hline & 冰草 A. cristatum & 6.46 & 4.19 & 88.47 & 35.78 & 200.54 & 130.13 & 8 \\
\hline & 阿尔泰狗娃 H. altaicus & 30.45 & 20.05 & 78.65 & 33.60 & 112.23 & 64.7 & 12 \\
\hline & 长芒草 $S$. bungeana & 20.47 & 13.88 & 79.46 & 25.66 & 160.27 & 90.52 & 11 \\
\hline & 中华隐子草 C. squarrosa & 30.51 & 10.42 & 50.26 & 25.09 & 76.12 & 40.56 & 12 \\
\hline & 白羊草 $B$. ischaemum & 30.55 & 22.37 & 53.63 & 28.32 & 69.46 & 35.04 & 8 \\
\hline 二级河阶地 & 无 No & & & 129.11 & 23.79 & 277.67 & 131.15 & 10 \\
\hline \multirow{6}{*}{$\begin{array}{l}\text { Lower river } \\
\text { terrace }\end{array}$} & 狗尾草 $S$. viridis & 10.14 & 3.22 & 120.15 & 26.75 & 246.5 & 128.62 & 9 \\
\hline & 猪毛蒿 A. scoparia & 156.30 & 75.28 & 88.49 & 36.76 & 38.99 & 20.18 & 12 \\
\hline & 冰草 A. cristatum & 8.46 & 5.18 & 110.35 & 26.03 & 213.85 & 140.49 & 9 \\
\hline & 阿尔泰狗娃 $H$. altaicus & 40.35 & 24.57 & 80.17 & 42.03 & 97.46 & 68.74 & 11 \\
\hline & 长芒草 $S$. bungeana & 20.20 & 10.69 & 96.44 & 26.35 & 195.86 & 103.33 & 12 \\
\hline & 中华隐子草 $C$. squarrosa & 36.90 & 20.48 & 39.45 & 27.26 & 57.81 & 39.68 & 8 \\
\hline
\end{tabular}

\section{2 不同立地条件下猪毛蒿种内、种间竞争}

猪毛蒿在有邻体生长时, 高度和地上生物量都 小于单独生长时的相应指标 (表 2), 说明 3 种立地 条件下各测试植物的种内、种间竞争都会影响到猪 毛蒿的高度和地上生物量。均值差异显著性检验表
明( Mann-Whitney $U$ 检验，表 3) 3 种立地条件下，狗 尾草、冰草和长芒草的种间竞争对猪毛蒿高度和地 上生物量的影响都不显著,阿尔泰狗娃花和中华隐 子草的种间竞争对目标种猪毛蒿高度和地上生物量 的影响都显著。白羊草在梁峁阴坡地和二级河阶地 
中对猪毛蒿的种间竞争也显著地影响到了目标种猪 毛蓄的高度和生物量。梁峁阴坡地和二级河阶地 中 种内竞争对目标种猪毛蒿高度和地上生物量影 响显著。在一级河阶地，种内竞争对猪毛蒿地上生 物量影响显著, 但对高度影响不显著。

由于各测试植物对猪毛蒿的竞争抑制程度是有 差别的(3 种立地条件下对高度和地上生物量为指 标的 RCI 经 Kruskal-Wallis 检验，其显著水平都远小 于 0.01)，从而形成了对猪毛蒿的竞争等级 (Competitive hierarchy)。以猪毛蒿在邻体竞争条件下生长量 的减小程度, 即 RCI 来看, 梁峁阴坡地各测试植物 对猪毛蒿高度的竞争等级是: 白羊草 > 中华隐子草 $>$ 猪毛蓠 $>$ 阿尔泰狗娃花 $>$ 长芒草 $>$ 狗尾草 $>$ 冰 草 对猪毛蒿地上生物量的竞争等级与上基本相同 (Spearman $R=0.857, p=0.014$ ) , 为猪毛蒿 $>$ 白羊草 $>$ 中华隐子草 $>$ 阿尔泰狗娃花 $>$ 长芒草 $>$ 冰草 $>$ 狗 尾草, 其它样地竞争等级类推。并且一、二级河阶地 中各测试植物对猪毛蒿高度与地上生物量的竞争等 级也无显著差别(Spearman $R$ 分别为 $0.857 、 0.829, p$ 分别为 $0.014 、 0.042)$, 说明猪毛蒿的生长形态虽然 会受到竞争的影响，但其高度与生物量仍有较强的 线形关系(图 2), 使得各测试植物对猪毛蒿高度与 地上生物量的竞争等级无显著区别。

不同立地条件下，种间竞争对猪毛蒿高度总的 受竞争抑制程度平均值来看, 以梁峁阴坡地最大, 其
次为二级河阶地，一级河阶地目标种猪毛蒿被竞争 抑制程度最小 (平均值分别为 $0.3837 、 0.2618$ 和 $0.2452)$ ，对猪毛蕎生物量总的受竞争抑制程度来 看, 以梁崩阴坡地较大, 而一、二级河阶较小(分别为 $0.4392 、 0.4264$ 和 0.4236$)$ 。在 3 种立地条件下各 测试植物单位重量对猪毛蒿的平均竞争抑制程度同 样以峁梁阴坡地最大, 其次为二级河阶地，一级河阶 地最小 (平均分别为 $0.0300 、 0.0229$ 和 0.0247 )。 说明立地条件越差, 资源可利用性越低, 种间竞争越 激烈，这与 Davies(1928)的结论一致。

对于猪毛蓠的种内竞争来说，因竞争效应是资 源捕获能力的反映和资源调节的结果, 而且同种植 物对土壤资源的竞争一般是大小对称 (Size symmetry)的 (Casper \& Jackson ,1997;Cahill \& Casper 2000)， 邻体猪毛蒿相对目标种猪毛蒿地上生物量越大(测 试植物猪毛蒿生物量与目标种猪毛蒿生物比值为： 梁峁阴坡地(14.62)>二级河阶地 (4.01) > 一级河 阶地(2.78)), 目标种受抑制程度越大, 因此目标种 猪毛蒿总的受竞争抑制程度为: 梁峁阴坡地 $>$ 二级 河阶地 >一级河阶地; 但从单位重量邻体猪毛蒿对 目标种猪毛蒿的竞争抑制来看, 则为一级河阶地 > 二级河阶地 > 梁峁阴坡地。说明立地条件越好, 目 标种猪毛蒿获得的资源相对其大小越少, 其受竞争 抑制程度越大，造成这种情况的可能原因是 :在一、 二级河阶地除土壤资源竞争外尚存在光资源竞争，

表 3 不同立地条件下各测试植物对猪毛蒿的相对竞争强度及其对猪毛蒿生长的影响

Table 3 The significance level of Artemisia scoparia traits under different test species' competition

\begin{tabular}{|c|c|c|c|c|c|c|}
\hline \multirow{2}{*}{$\begin{array}{l}\text { 地点 } \\
\text { Sites }\end{array}$} & \multirow{2}{*}{$\begin{array}{l}\text { 测试植物 } \\
\text { Test species }\end{array}$} & \multicolumn{2}{|c|}{ 高度 Height } & \multicolumn{3}{|c|}{ 地上生物量 Biomass above ground } \\
\hline & & $P_{U}$ & $R C I_{T_{\text {otal }}}^{H}$ & $P_{U}$ & $R C I_{\text {Total }}^{H}$ & $R C I_{\text {Pergram }}$ \\
\hline 梁峁阴坡地 & 狗尾草 Setaria viridis & 0.4912 & 0.0632 & 0.6166 & 0.0670 & 0.0108 \\
\hline Northern mound & 猪毛蒿 Artemisia scoparia & 0.0001 & 0.5440 & 0.0001 & 0.9436 & 0.0045 \\
\hline \multirow[t]{5}{*}{ land } & 冰草 Agropyron cristatum & 0.7395 & 0.0230 & 0.1063 & 0.1667 & 0.0748 \\
\hline & 阿尔泰狗娃 Heteroppus altaicus & 0.0001 & 0.2824 & 0.0001 & 0.5410 & 0.0166 \\
\hline & 长芒草 Stipa bungeana & 0.0687 & 0.1318 & 0.0629 & 0.2571 & 0.0471 \\
\hline & 中华隐子草 Cleistogenes squarrosa & 0.0001 & 0.5801 & 0.0001 & 0.7650 & 0.0180 \\
\hline & 白羊草 Bothriochloa ischaemum & 0.0001 & 0.6214 & 0.0001 & 0.8386 & 0.0124 \\
\hline 一级河阶地 & 狗尾草 $S$. viridis & 0.8245 & 0.0294 & 0.9602 & 0.0128 & 0.0036 \\
\hline The lowest river & 猪毛蒿 A. scoparia & 0.1544 & 0.2217 & 0.0008 & 0.8011 & 0.0060 \\
\hline \multirow[t]{5}{*}{ terrace } & 冰草 $A$. cristatum & 0.8392 & 0.0337 & 0.5318 & 0.1754 & 0.0271 \\
\hline & 阿尔泰狗娃 $H$. altaicus & 0.3353 & 0.1410 & 0.0147 & 0.5385 & 0.0177 \\
\hline & 长芒草 $S$. bungeana & 0.3241 & 0.1322 & 0.1935 & 0.3410 & 0.0167 \\
\hline & 中华隐子草 $C$. squarrosa & 0.0016 & 0.4511 & 0.0026 & 0.6870 & 0.0225 \\
\hline & 白羊草 B. ischaemum & 0.0127 & 0.4143 & 0.0020 & 0.7144 & 0.0234 \\
\hline 二级河阶地 & 狗尾草 $S$. viridis & 0.4501 & 0.0694 & 0.7158 & 0.1123 & 0.0111 \\
\hline Lower river & 猪毛蒿 A. scoparia & 0.0315 & 0.3146 & 0.0031 & 0.8596 & 0.0055 \\
\hline \multirow[t]{4}{*}{ terrace } & 冰草 $A$. cristatum & 0.4538 & 0.1453 & 0.4829 & 0.2298 & 0.0272 \\
\hline & 阿尔泰狗娃 $H$. altaicus & 0.0043 & 0.3791 & 0.0175 & 0.6490 & 0.0161 \\
\hline & 长芒草 $S$. bungeana & 0.1047 & 0.2530 & 0.2568 & 0.2946 & 0.0146 \\
\hline & 中华隐子草 C. squarrosa & 0.0001 & 0.6944 & 0.0048 & 0.7918 & 0.0215 \\
\hline
\end{tabular}


而光资源竞争是不对称 (Size asymmetry) 的 (Rees \& Bergelson ,1997) 即测试植物猪毛蓠中相对较大个体 获得了相对其大小更多的光资源，对目标种猪毛蕎 造成了遮阴条件。单位重量竞争能力排除了邻体大 小对猪毛蕎竞争抑制程度的影响, 是测试植物相对 目标种竞争能力的真实反映, 根据该指标来看, 立地 条件越好 猪毛蒿种内竞争抑制效应越大。

不同立地条件下每种测试植物对猪毛蕎的相对 竞争能力 $\left(R C I_{\text {Pergram }}\right)$ 也有所不同，从而影响到了各 测试植物对猪毛蕎的竞争等级, Spearman 秩相关检 验表明 :各测试植物在一级河阶地与梁峁阴坡地对 猪毛蒿的竞争等级存在显著差别 $(R=0.8216, R=$ $0.0263)$, 而其它立地条件下竞争等级差异不显著。 说明立地条件差异较大时，植物的竞争等级会发生 显著变化。从表 3 可以看出,3种立地条件下对猪 毛蓄相对竞争能力较强的植物都是演替后期种, 生 活型为多年生草本, 特别是根茎型禾草和丛生型禾 草对猪毛蕎的相对竞争能力较强, 而一、二年生植物 对猪毛蒿相对竞争能力较弱。

\section{3 结论与讨论}

植物竞争是影响群落结构与动态的重要因素之 一, 同时植物种的竞争能力还受到许多生物和非生 物因素的影响(李博,2001;杜峰等,2004)。已有研 究表明:立地条件如土壤养分、水分资源等对植物生 长、竞争都有较为重要的影响 (Wichmann,2001) , 进 而会影响到群落结构与动态 ( Tilman, 1982;Crawley \& May ,1987 ; Rees \& Bergelson , 1997 ; Keddy et al. , 2002)。从本文结果来看, 可以初步得出以下结论 :

1) 河阶地和梁峛阴坡地中猪毛茣的生长都趋于 小型化, 有少数大个体和多数小个体。两种立地条 件下猪毛蒿地上部分都存在非线形异速生长, 竞争 对猪毛蒿地上部分生长存在塑造作用。河阶地猪毛 蒿高度和生物量变异性较梁峁阴坡地大。

由于种内或种间竞争的存在,植物群落内个体 的生长多趋于小型化,但一旦竞争引起自疏或他疏 时，小型化趋势会有所减小(李博，2001)。应该说明 的是本文只比较了不同立地条件下猪毛蒿的生长特 征 对于自然条件下猪毛蓄是否会发生自疏或他疏 并未进行调查研究, 需进行生长动态和生命表等的 调查。河阶地与梁峁阴坡地高度和生物量的变异性 差异可能与资源竞争种类及群落密度有关: 河阶地 中因水肥条件较好(表 1),群落盖度较大，除土壤资 源竞争外尚存在着对光资源的竞争, 导致变异性相
对较大。而梁峁阴坡地群落盖度较小, 主要是对水 分和养分的竞争，不存在或很少存在对光的竞争 地 上生物量的变异性主要是猪毛蒿本身生长速率差异

(Turner \& Rabinnowitz ,1983 ;Weiner \& Thomas ,1986)、 萌发早晚的差别 (Spillards,1989;Firbank \& Watkinson ,1990) 和对水肥竞争造成的。河阶地和梁峁阴 坡地猪毛蒿大小变异性的差异在一定程度上支持了 优势-压抑假说 (Dominance-suppression hypothesis) (Turner \& Rabinnowitz,1983;Weiner \& Thomas ,1986) , 即在拥挤的种群或群落中, 大小变异性更大。在立 地条件较差时 猪毛蒿的种内竞争主要是对土壤资 源的竞争, 是大小对称的, 特别是在密度较小的时 候;而在立地条件较好时,除对土壤资源的竞争外， 还有对光资源的竞争，较好的立地条件增加了大个 体在光资源竞争中的有利地位, 种内竞争是大小不 对称的（Wichmann，2001），在不对称竞争的情况下， 群落内大个体获得了相对于其大小更多的资源, 这 可能是河阶地猪毛蕎大小变异性较梁峛阴坡地大的 原因。

2)多数竞争试验以测试植物对目标种总的竞争 抑制程度来表示测试植物相对目标种的竞争能力。 从猪毛蕎总的受竞争抑制程度来看, 因测试植物猪 毛蒿生物量相对较高，对目标种生物量抑制程度最 大 ;白羊草、中华隐子草和阿尔泰狗娃花等测试植物 虽然生物量较小，但其对猪毛蒿的竞争抑制程度也 相对较大，而一年生植物狗尾草则较小，这说明植物 的竞争能力主要与种的生态学特性有关, 另外还会 受到个体大小的影响。因此用 $R C I_{\text {Total }}$ 表示植物的 相对竞争能力在同一立地条件下是可行的,但在不 同立地条件下测试植物大小，如生物量等会发生较 大变化, 会影响到测试植物相对竞争能力的准确评 价。因此本研究同时采用单位重量竞争强度这一指 标。从这一指标来看, 多年生植物单位地上生物量 对猪毛蓠的相对竞争能力较一、二年生植物强, 再一 次说明演替后期种对前期种的竞争是推动演替发生 的机制之一。按照演替生态位理论, 演替后期物种 应该比演替前期物种具有更强的竞争能力, 表 2 和 表 3 的结果也证实了这一点。

单位重量竞争能力与总体竞争能力这一指标相 比, 其生态学意义更加明确。因植物的竞争能力决 定于资源获得能力或忍耐低资源水平的能力, 如果 小个体与大的邻体相比具有较大的单位重量资源吸 收率, 也就是单位重量竞争能力强, 从长远来说是有 利的，因为它获得了相对于其个体大小更多的资源。 
Miller 和 Werner ( 1987) 发现虽然植物之间的单位重 量的竞争能力差别比较小, 但群落中各种植物的这 种单位重量的竞争能力排序与相对多度密切相关。 Howard(2001) 的结果也证实:单位重量竞争能力排 序与种的相对多度排序关系更密切, 比总竞争能力 更能说明自然条件下种的相对多度。本研究从种间 竞争角度研究了黄土丘陵区猪毛蒿群落的演替方向 和机制，即黄土丘陵区农田摞荒后的猪毛蓠单优群 落或共优群落, 为冰草群落或阿尔泰狗娃花群落或 长芒草群落代替的原因。研究结果显示: 白羊草、中 华隐子草、冰草、长芒草和阿尔泰狗娃花等演替后期 种与演替前期种狗尾草和猪毛蒿相比, 对演替前期 群落优势种猪毛蒿有相对较大的竞争优势, 使得猪 毛蕎个体出现小型化趋势, 逐渐演替为其它群落。

多数植物间的竞争具有不对称性,进而形成了 竞争等级。竞争等级的表达同样还受生物和非生物 条件的影响，如群落动态、资源可利用水平、生态位 重叠程度和现实生态位与最适生态位的偏移程度 等。因此植物竞争过程与环境条件有关, 其竞争结 果也就会受到环境条件的影响 (Sharitz \& McCormick , 1973 ;Grace , 1989 ;Keddy et al. ,2002), 但 这种影响, 是否足以改变种的相对竞争能力以至竞 争等级, 尚无定论, 已有研究中植物竞争等级在不同 环境条件下发生变化与未发生变化的比例大约各一 半 (Goldberg ,1996) 这可能与环境条件的差别有关。 从本研究来看,一级河阶地和梁峁阴坡地各测试植 物对猪毛蒿的竞争等级发生了变化, 这说明环境条 件差别较大时 植物的竞争等级会发生显著变化。

\section{参 考 文 献}

Aerts R (1999). Interspecfic competition in natural plant communities: mechanisms, trade-offs and plant-soil feedbacks. Journal of Experimental Botany, 50, 29-37.

Aguiar MR, Lauenroth WK, Peters DP (2001). Intensity of intraand interspecific competition in coexisting shortgrass species. Journal of Ecology, 89, 40-47.

Begon M, Harper JL, Townsend CR (1990) . Ecology, Individuals, Populations and Communities 2nd edn. Blackwell Scientific Publications, Oxford.

Cahill JF, Casper BB (2000). Investigating the relationship between neighbor root biomass and belowground competition: field evidence for symmetric competition belowground. Oikos, 90, 311 -320 .

Casper BB, Jackson RB (1997). Plant competition underground. Annual Review of Ecology and Systematics, 28, 545 - 570 .

Cheng ZH (2001). Growth and Competition of Fourteen Herb species in Experimental Microcoenosiums. Acta Botanica Sinica (植物学报), 43, $1184-1190$.

Crawley MJ, May RM(1987). Population dynamics and plant community structure: competition between annuals and perennials. Journal of Theoretical Biology, 125, 475-489.

Davies W (1928). The factor of competition between one species and another in seeds mixtures. In: Welsh Plant Breeding Station ed. Bulletin H8. Aberystwyth, 82-149.

Du F(杜峰), Liang ZS(梁宗锁), Hu LJ (胡莉娟) (2004)。A reviews on plant competition. Chinese Journal of Ecology (生态 学杂志), 23 (4), 157 - 163. (in Chinese with English abstract)

Firbank LG, Watkinson AR(1990). On the effects of competition: from monocultures to mixtures. In: Grace JB, Tilman D eds. Perspectives on Plant Competition. Academic Press, New York, $165-192$.

Fowler N (1986). The role of competition in plant communities in arid and semiarid regions. Annual Review of Ecology and Systematics, 17, 89-110.

Goldberg DE (1990). Components of resources in plant communities. In: Grace JB, Tilman D eds. Perspectives on the Plant Competition. Academic Press, New York, $27-49$.

Goldberg DE (1996). Competitive ability: definitions contingency and correlated traits. Philosophical Transactions of the Royal Society of London B, 351, 1377 - 1385.

Grace JB (1989). Effects of water depth on Typha latifolia and Typha domingensis. American Journal of Botany, 76, $762-768$.

Grace JB (1995). On the measurement of plant competition intensity. Ecology, 76, $305-308$.

Hamilton JG, Holzapfel C, Mahall BE (1999). Coexistence and interference between a native perennial grass and non-native annaul grasses in California. Oecologia, 121, 518-526.

Howard TG (2001). The relationship of total and per-gram rankings in competitive effect to the natural abundance of herbaceous perennials. Journal of Ecology, 89, 110-117.

Keddy P, Nielsen K, Weiher E, Lawson R (2002) . Relative competitive performance of 63 species of terrestrial herbaceous plants. Journal of Vegetation Science, 13, 5-16.

Leuschner C, Hertel D, Coners H, Büttner V (2001). Root competition between beech an oak: a hypothesis. Oecologia, 126, $276-284$.

Li B(李博), Chen JK(陈家宽), Watkinson AR (1998). A literature review on plant competition. Chinese Buttetin of Botany (植 物学通报), 15(4), 18-29. (in Chinese with English abstract)

Li B (李博) (2001). Plant Competition-Studies on the Interactions Between Crop and Weed. Higher Education Press and Springer Press, Beijing, 251. (in Chinese)

Li ZF(李仲芳), Wang G(王刚) (2002). The effects of intraspecific competition on the relationship between height growth and biomass of annual plants. Journal of Lanzhou University (Natural 
Sciences) (兰州大学学报 (自然科学版)), 38 (2), 141 146. (in Chinese with English abstract)

Martin RA, William KL, Debrap PP (2001). Intensity of intra- and interspecific competition in coexisting shortgrass species. Journal of Ecology, 89, $40-47$.

Miller TE, Werner PA(1987) . Competitive effects and response between plant species in a first year old-field community. Ecology, $68,1201-1210$.

Negbi M, Evenari M(1961). The means of survival of some desert summer annuals. In: Plant-Water Relationships in Arid and Semiarid Conditions. UNESCO, Paris, 249.

Rees M, Bergelson J (1997). Asymmetric light competition and founder control in plant communities. Journal of Theoretical Biology, 184, $353-358$.

Rees M, Condit R, Crawley M, Pacala S, Tilman D(2001) . Longterm studies of vegetation dynamics. Science, 293, 650-655.

Sharitz RR, McCormick JF ( 1973 ). Population dynamics of two competition plant annual species. Ecology, 54, 723 - 740 .

Spillards DM (1989). Studies of Plant Competition. PhD dissertation, University of East Anglia, Norwic.

Tang QY (唐启义)，Feng MG (冯明光) (1997)。 Practical Statistics and DPS Data Processing System. China Agricultural Press, Beijing, 407. (in Chinese)

Tielbörger K, Kadmon R (2000). Indirect effects in a desert plant community: is competition among annuals more intense under shrub canopies? Plant Ecology, 150, 53 - 63.

Tilman D(1982). Resource Competition and Community Structure. Princeton University Press, Princeton, New Jersey.
Tilman D(1988) . Plant Strategies and the Dynamics and the Structure of Plant Communities. Princeton University Press, Princeton, New Jersey.

Turner MD, Rabinowitz D(1983). Factors affecting frequency distributions of plant mass: the absence of dominance and suppression in competing monocultures of Festuca paradoxa. Ecology, $64,469-475$.

Wang ZQ (王政权), Wu GS (吴巩胜), Wang JB (王军邦) (2000). Application of competition index in assessing intraspecfic and interspecfic spatial relations between manchurian ash and dahurian larch. Chinese Journal of Applied Ecology (应用生态学 报), 11, 641 - 645. ( in Chinese with English abstract)

Weiner J, Thomas SC (1986). Size variability and competition in plant monocultures. Oikos, 47,211-222.

Weiner J, Thomas SC (1992) . Competition and allometry in three species of annual plants. Ecology, 73, 648-656.

Wichmann L(2001). Annual variations in competition symmetry in even-aged sitka spruce. Annals of Botany, 88, 145 - 151 .

Zhang ZP(张泽浦), Fang JY (方精云), Kan M(2000). Effects of competition on growth rate and probability of death of plant individuals: a study based on nursery experiments of Larix leptolepis populations. Acta Phytoecologica Sinica (植物生态学 报), 24, 340-345. (in Chinese with English abstract)

Zou CJ(刍䐏静)，Xu WD(徐文铎) (1998). Study on intraspecific and interspecfic competition of Picea mongolica. Acta Phytoecologfca Sinica (植物生态学报), 22, 269-274. (in Chinese with English abstract) 